Manuelle Medizin 2019·57:16-20 https://doi.org/10.1007/s00337-018-0490-6 Online publiziert: 15. Januar 2019

(c) Der/die Autor(en) 2019

CrossMark

Stefan Salminger · Johannes A. Mayer · Agnes Sturma · Oskar C. Aszmann

Klinische Abteilung für Plastische und Rekonstruktive Chirurgie, Christian Doppler Labor für Wiederherstellung von Extremitätenfunktionen Universitätsklinik für Chirurgie, Medizinische Universität Wien, Wien, Österreich

\title{
Prothetischer Ersatz an der oberen Extremität bei Amputation oder Funktionsverlust
}

Im vorliegenden Beitrag werden die Möglichkeiten des prothetischen Ersatzes bei Amputationen sowie funktionsloser Hand dargestellt.

\section{Funktionslose obere Extremität}

Schwerwiegende Knochen- oder Weichteilverletzungen der oberen Extremität müssen zwar notwendigerweise zu einer Amputation der Hand oder des Arms führen, können allerdings einen schwerwiegenden Funktionsverlust nach sich ziehen. Dies gilt z. B. für ausgeprägte Verletzungen des Armnervengeflechts, aber auch für Verbrennungs-, Explosionsoder Avulsionstraumata [2]. Trotz adäquater und zeitgerechter chirurgischer Rekonstruktion kann in manchen Fällen keine sinnvolle Extremitätenfunktion wiederhergestellt werden [4]. In diesen Situationen hat der prothetische Ersatz in den letzten Jahren einen immer höheren Stellenwert erlangt [2]. Trotzdem ist es wichtig festzuhalten, dass der prothetische Ersatz mit einer suffizienten biologischen Rekonstruktion nicht konkurrieren kann und soll. Er bietet allerdings eine sinnvolle Alternative, um am Ende der biologischen rekonstruktiven Leiter eine Wiederherstellung einer Extremitätenfunktion zu ermöglichen [2]. In diesen Fällen dient die Prothese als Rekonstruktion einer Hilfshand bzw. eines Hilfsarms, um den Patienten im täglichen Leben und bei der Arbeit zu unterstützen.
Eine nicht funktionstüchtige Hand bzw. einen funktionslosen Arm mit einem Stück Technik zu ersetzen, stellt einen neuen Ansatz in der Extremitätenrekonstruktion dar. Die notwendige elektive Amputation inklusive möglicher Nerventransfers oder Muskeltransplantationen zur Schaffung von Muskelsignalen und Ersatz mit myoelektrischer Prothese wird als bionische Rekonstruktion bezeichnet [2]. Essenziell hierbei ist die adäquate Indikationsstellung.

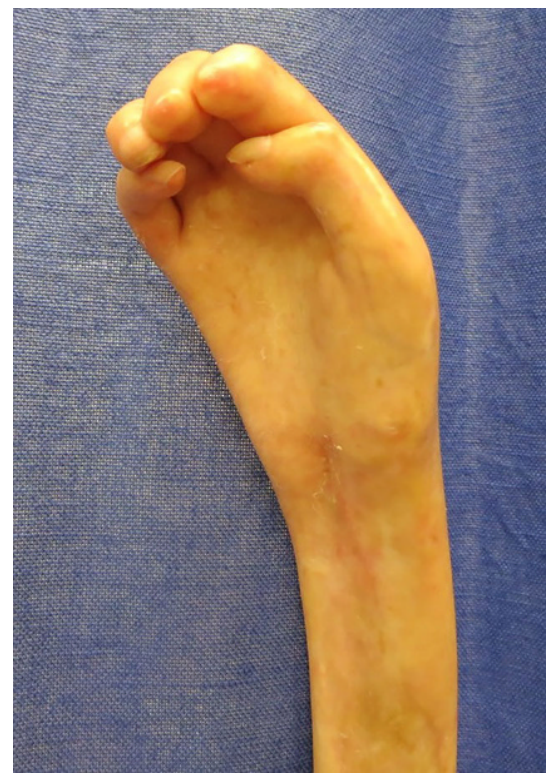

Abb. 1 ॥ Funktionslose Hand nach Kompartmentsyndrom 


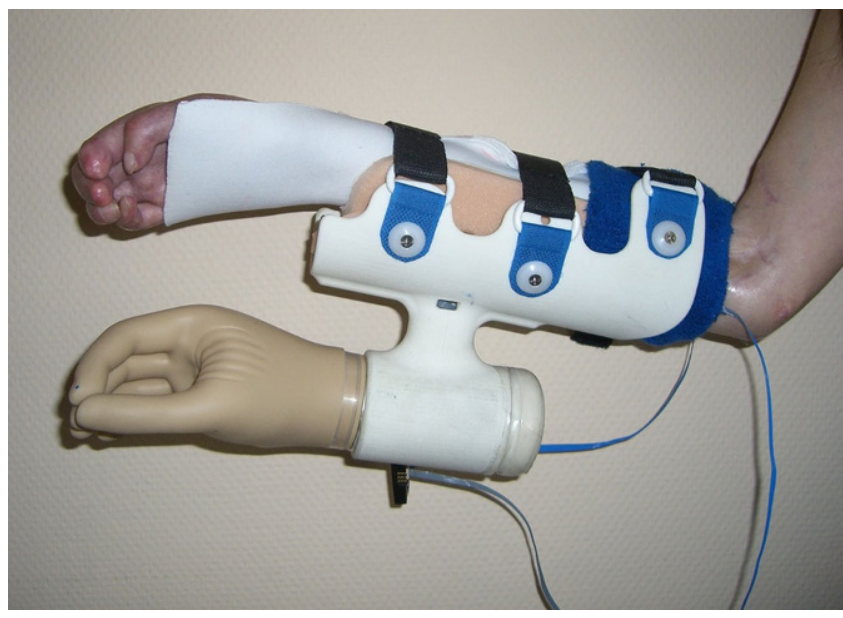

Abb. $2 \Delta$ Versorgung mit Hybridprothese

\section{Prothetische Rekonstruktion}

Im Folgenden wird anhand eines Patientenbeispiels der Prozess der prothetischen Rekonstruktion bei funktionsloser Extremität dargestellt.

Bei der Patientin handelt es sich um eine 29 Jahre alte Frau, die im Rahmen der langwierigen Behandlung eines ursprünglich gutartigen Knochentumors von Radius und Ulna ein Kompartmentsyndrom entwickelte. Trotz zeitnaher Fasziotomie und nach einer zusätzlichen bakteriellen Infektion kam es zu einer Volkmann-Kontraktur und nach langer Ruhigstellung $\mathrm{zu}$ einer „frozen hand". Selbst mit intensiver Ergo- und Physiotherapie konnte keine brauchbare Handfunktion wiedererlangt werden. Die Ellenbogenbeweglichkeit war auf ein Minimum eingeschränkt und die Hand größtenteils asensibel, dystroph und für die Patientin funktionslos (• Abb. 1). Eine biologische Rekonstruktion war aufgrund der atrophen und durch das Kompartmentsyndrom schwer geschädigten Unterarmmuskulatur nicht möglich.

Zur Austestung und Veranschaulichung der prothetischen Möglichkeiten wurde die Patientin mit einer sog. Hybridprothese provisorisch versorgt. Hierfür wird eine myoelektrische Prothese mithilfe einer Schienenkonstruktion an die existierende Hand montiert, um die vorhandenen Myosignale zu testen und zu trainieren sowie dem Patienten die zu erwartende prothetische Funktion vor

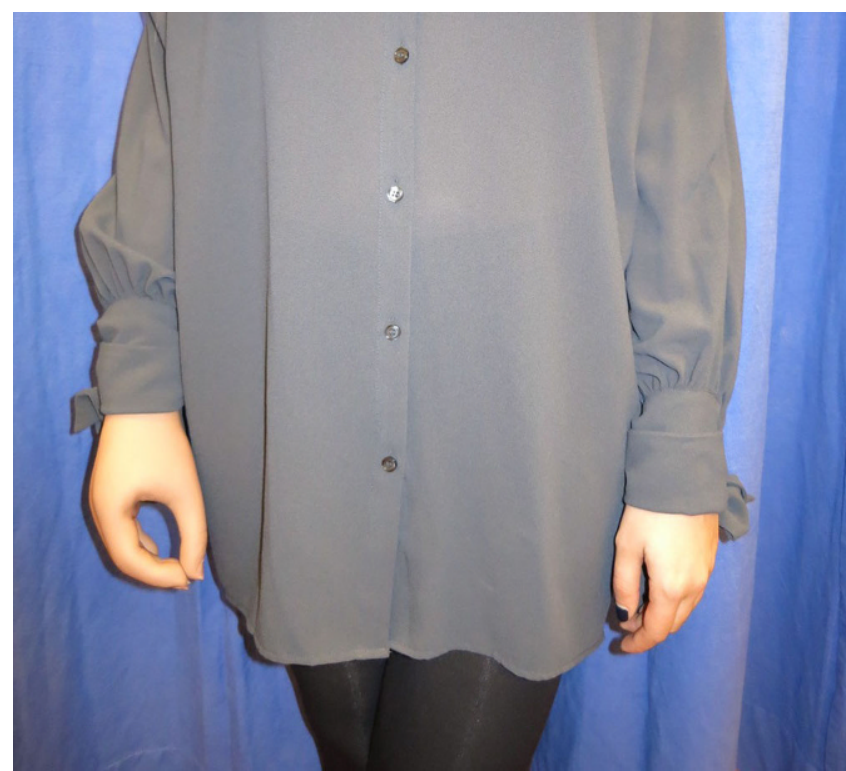

Abb. $3 \Delta$ Zustand nach erfolgter Prothesenanpassung

Augen zu führen (• Abb. 2). Dies stellt einen wichtigen Schritt in der Entscheidungsfindung sowohl für den Patienten als auch für den Arzt dar. Nimmt der Patient einen deutlichen Funktionsgewinn wahr und ist dieser auch mit einem objektiven Handfunktionstest belegbar, können eine elektive Amputation und ein prothetischer Ersatz auf Wunsch des Patienten geplant werden. Präoperativ werden alle potenziellen Patienten einer psychologischen Evaluation unterzogen, um Coping-Mechanismen entsprechend abzuklären.

Nach erfolgreicher Anpassung der Hybridprothese entschied sich die Patientin für eine bionische Rekonstruktion und daher für eine elektive transradiale Amputation. Im Rahmen der Amputation wurde eine extensive Arthrolyse des Ellenbogengelenks durchgeführt. Der Bewegungsumfang im Ellenbogengelenk konnte dadurch deutlich verbessert und das Extensionsdefizit auf $20^{\circ}$ reduziert werden. Aufgrund des dystrophen Zustands des Unterarms und der multiplen Voroperationen kam es postoperativ zu einer verzögerten Wundheilung. Acht Wochen nach Amputation konnte mit der Prothesenversorgung begonnen werden. Die myoelektrische Prothese wurde mit 2 Muskelsignalen, an den Extensoren und an den Flexoren, gesteuert. Die bionische Rekonstruktion ermöglichte dieser jungen Patientin nicht nur funktionell, sondern auch ästhetisch eine deutliche Verbesserung (• Abb. 3).

Aufgrund dieser Ergebnisse wurde das Konzept der bionischen Rekonstruktion auch bei Patienten mit angeborenen Extremitätenfehlbildungen angewandt [13]. Es kann auch bei Patienten mit Nervenausrissverletzungen die oft enorm quälenden Deafferenzierungsschmerzen, die aufgrund der inneren nervalen Amputation zustande kommen, deutlich reduzieren [4]. Hierbei dürfte die prothetische Versorgung als eine kontinuierliche Spiegeltherapie und funktionelle Rückkoppelung dienen und dadurch die Schmerzsituation verbessern.

\section{Hohe Amputation}

Konventionelle myoelektrische Prothesen werden mit 2 Muskelsignalen, die von zwei getrennt innervierten Muskelgruppen der verbliebenen Stumpfmuskulatur stammen, angesteuert. Auf transhumeralem Amputationsniveau werden der M. biceps und M. triceps, bei glenohumeralen Stümpfen meist der M. pectoralis major und der M. latissimus dorsi als Signalgeber verwendet. Die einzelnen prothetischen Gelenke und Funktionen (Hand, Handgelenk, Ellenbogen) müssen mittels unterschiedlicher Algorith- 
men, wie z.B. Kokontraktionen der erwähnten beiden Muskeln, angewählt und in der jeweiligen Ebene mit denselben Muskeln linear angesteuert werden. Somit sind mit ein und derselben kognitiven „Bewegung“ verschiedene prothetische Funktionen auszuüben. Ein Patient mit transhumeraler Amputation muss daher mit dem M. biceps, kognitiv Ellenbogenbeugung, nicht nur den prothetischen Ellenbogen beugen, sondern nach Umschalten auch die Hand schließen und das Handgelenk drehen. Ein harmonischer, dem natürlichen Bewegungsmuster einigermaßen entsprechender Bewegungsablauf ist mit diesem Steuerungsmechanismus nicht möglich.

\section{Selektive Nerventransfers}

Die chirurgische Multiplikation von Muskelsignalen zur Verbesserung der Prothesensteuerung wurde erstmals von Kuiken et al. [5] beschrieben. Hierbei werden die Nerven, die durch die Amputation ihr Zielorgan verloren haben, im Wesentlichen N. radialis, N. medianus, $\mathrm{N}$. ulnaris und bei glenohumeraler Amputation auch der N. musculocutaneus, an einzelne Muskeln im Stumpfbereich transferiert [11]. Nach erfolgter Nervenregeneration ist es dadurch möglich, eine suffiziente Anzahl intuitiv steuerbarer Muskelsignale zu kreieren. Diese Technik der selektiven Nerventransfers bei Amputierten wird "targeted muscle reinnervation“ (TMR) genannt. Je nach Amputationshöhe hat sich ein bestimmtes Nerventransferschema etabliert ([11]; - Tab. 1 und 2) Die Nerven, die ihr Zielorgan im Rahmen der Amputation verloren haben, werden möglichst muskelnah an den selektiven Nervenast des jeweiligen Zielmuskels transferiert, hierfür wird der Zielmuskel naturgemäß für die Dauer der Reinnervation denerviert. Nach erfolgter Reinnervation spannen sich schließlich die Zielmuskeln entsprechend der Aktivität der Spendernerven an und ihr Muskelpotenzial kann mithilfe von Oberflächenelektroden zur Prothesensteuerung verwendet werden. Ziel dieser Operation ist es, den Patienten mit bis zu 6 individuellen Muskelsignalen auszustatten. Auf diese Weise ist eine harmonische, intuitive, dem natür-

Manuelle Medizin 2019·57:16-20 https://doi.org/10.1007/s00337-018-0490-6

(c) Der/die Autor(en) 2019

S. Salminger · J. A. Mayer · A. Sturma - O. C. Aszmann

\section{Prothetischer Ersatz an der oberen Extremität bei Amputation oder Funktionsverlust}

\section{Zusammenfassung}

Hintergrund. Die Indikation zum prothetischen Ersatz der oberen Extremität wurde in den letzten Jahren von der klassischen Amputation auch auf Patienten mit Verlust oder angeborenen Defiziten der Hand- und Armfunktion ausgeweitet. Des Weiteren wurde die Steuerung myoelektrischer Hand- und Armprothesen mittels selektiver Nerventransfers im Stumpfbereich deutlich verbessert.

Fragestellung. Übersicht über die chirurgischen, therapeutischen und prothetischen Möglichkeiten bei Amputationen oder Funktionsverlust der oberen Extremität. Material und Methode. Es erfolgte eine selektive Literaturrecherche unter Berücksichtigung eigener Erfahrungen des klinischen Alltags und Durchsicht von Patientenakten. Ergebnisse. Die elektive Amputation mit prothetischem Ersatz führt bei Patienten mit funktionsloser Hand zu einer deutlichen Verbesserung der Hand- und Armfunktion und zeigt dadurch auch substanzielle Verbesserungen der Lebensqualität in diesem speziellen Patientengut. Im Falle einer hohen Amputation ermöglichen selektive Transfers der Nerven des Plexus brachialis auf die verbliebene Stumpfmuskulatur die Etablierung von bis zu 6 Signalgebern, wodurch intuitiv und simultan die verschiedenen prothetischen Gelenke gesteuert werden können. Auf diese Weise ist eine deutlich effizientere Steuerung möglich, ohne zwischen den verschiedenen Steuerungsebenen der Prothese wechseln zu müssen.

Schlussfolgerung. Der prothetische Ersatz der oberen Extremität stellt nicht nur den Goldstandard nach Amputationen dar, sondern ermöglicht auch für Patienten mit funktionsloser Hand aufgrund massiver Weichteil- oder Nervenverletzung sowie angeborener Defekte eine funktionelle Wiederherstellung.

Schlüsselwörter

Hand · Neurom · Nerventransfer .

Rekonstruktion · Rehabilitation

\section{Prosthetic replacement of the upper extremity after amputation or loss of function}

Abstract

Background. The indication for prosthetic replacement of the upper extremity could be extended in recent years from classic amputation to patients suffering loss or congenital deficits of hand and arm function. Additionally, the control of myoelectric hand and arm prostheses could be improved by the use of selective nerve transfers.

Objectives. Overview regarding surgical, therapeutical and prosthetic options after amputations or loss of function of the upper extremity.

Methods. Selective literature search including the authors' own experience in everyday clinical practice as well as a review of medical records.

Results. Elective amputation in combination with prosthetic replacement in patients after loss of hand function can achieve remarkable improvements in hand and arm function, as well as quality of life in this special patient cohort. In cases of high-level amputations selective nerve transfers of the brachial plexus to remaining stump muscles can establish up to six myosignals to achieve intuitive and simultaneous control of the different prosthetic functions. In this way, more efficient control of the prosthetic device is possible without the need to change between the different artificial joints.

Conclusion. The prosthetic replacement of the upper extremity does not only represent the gold standard for amputees, but also offers useful reconstruction for patients suffering loss of hand and arm function after massive soft tissue or nerve trauma as well as congenital deficiencies.

Keywords

Hand $\cdot$ Neuroma $\cdot$ Nerve transfer . Reconstruction $\cdot$ Rehabilitation 
Tab. 1 Schema Nerventransfer nach transhumeraler Amputation

\begin{tabular}{|c|c|c|}
\hline Zielmuskeln/Signalgeber & Nerv & Prothesenfunktion \\
\hline M. biceps caput longum & N. musculocutaneus & Ellenbogenflexion \\
\hline M. biceps caput breve & N. ulnaris & Hand schließen \\
\hline M. brachialis & N. medianus & Pronation \\
\hline M. triceps caput longum/mediale & N. radialis & Ellenbogenextension \\
\hline M. triceps caput laterale & $\begin{array}{l}\text { Geteilter Ramus profundus } \\
\text { N. radialis }\end{array}$ & Hand öffnen \\
\hline M. brachioradialis & $\begin{array}{l}\text { Geteilter Ramus profundus } \\
\text { N. radialis }\end{array}$ & Supination \\
\hline
\end{tabular}

Tab. 2 Schema Nerventransfer nach glenohumeraler Amputation

\begin{tabular}{|c|c|c|}
\hline Zielmuskeln/Signalgeber & Nerv & Prothesen Funktion \\
\hline M. pectoralis major Pars clavicularis & N. musculocutaneus & Ellenbogenflexion \\
\hline M. pectoralis minor & N. ulnaris & Hand schließen \\
\hline M. pectoralis major Pars sternocostalis & N. medianus & Umwendbewegung \\
\hline M. pectoralis major Pars abdominalis & N. medianus & Umwendbewegung \\
\hline M. latissimus dorsi & N. radialis & Ellenbogenextension \\
\hline M. infraspinatus & $\begin{array}{l}\text { Ramus profundus } \\
\text { N. radialis }\end{array}$ & Hand öffnen \\
\hline
\end{tabular}

lichen Bewegungsmuster entsprechende Steuerung gewährleistet, ohne dass der Patient zwischen den verschiedenen Steuerungsebenen wechseln muss.

Voraussetzung für die Durchführung selektiver Nerventransfers sind intakte Muskeln im Bereich des Amputationsstumpfs sowie ein weitgehend intaktes proximales Armnervengeflecht. Diesbezüglich ist eine präoperative Magnetresonanztomographie, hochauflösender Ultraschall sowie Messung der Nervenleitgeschwindigkeit und Elektromyographie (EMG) des verbliebenen Armnervengeflechts zu empfehlen. Des Weiteren muss dem Patienten bewusst sein, dass nach erfolgter Operation und abgeschlossener Nervenregeneration ein intensives Signal- und Prothesentraining notwendig ist. Somit ist mit einer abgeschlossenen TMR-Prothesen-Versorgung erst etwa 12 Monate nach Operation zu rechnen.

\section{Nachbehandlung}

Die Nachbehandlung von Patienten mit TMR-Prothese hat sich als mindestens so entscheidend wie die Operation selbst herausgestellt. Der Patient muss unter gezielter Anleitung eines Therapeuten die Ansteuerung der einzelnen Muskelsignale erlernen und trainieren. Verschiedene these erst nach Reinnervation der ersten Muskelsignale wieder steuern.

Die Technik der selektiven Nerventransfers hat sich mittlerweile auch als solide Methode zur Behandlung von Neurom- bzw. Phantomschmerzen etabliert [15]. Denn schon ein einzelnes hypersensibles Neurom am Amputationsstumpf kann das Tragen einer Prothese unmöglich machen [1]. Selektive Nerventransfers, wie sie bei einer TMR-Operation durchgeführt werden, stellen eine sinnvolle Verbindung des betroffenen Nervs $\mathrm{zu}$ einem funktionellen Endorgan her. Dadurch erhält das zentrale Nervensystem wieder eine sinnvolle Rückmeldung, die schmerzhafte Lücke kann geschlossen werden und es kommt zu keiner erneuten Neurombildung. Dieklinische Erfahrung hat auch gezeigt, dass durch die verbesserte und intuitivere Prothesensteuerung und dadurch erhöhte Akzeptanz und Inkorporation der Prothese auch eine Reduktion der Phantomschmerzen zur erzielen ist. Dem Gehirn wird, ähnlich dem Prinzip der Spiegeltherapie, durch die Prothese eine sensorische wie auch visuelle Rückmeldung geboten und somit eine funktionstüchtige Extremität simuliert.

\section{Zukünftige Weiter- entwicklungen}

Myoelektrische Signale werden derzeit mittels Oberflächenelektroden aufgenommen und an die Prothese zur Steuerung weitergeleitet. Aufgrund von Weichteilverschiebungen über einem Muskel, der variablen Elektroden- bzw. Stumpfposition im Schaft, Kontaktverlust durch Hebelwirkungen und der Impedanzschwankungen je nach Temperatur und Transpiration der Haut kann es zu einer mangelnden Signalübertragung und im Weiteren zu Fehlfunktionen der Prothese kommen. Gegenstand laufender Studien sind implantierbare Elektroden. Hiermit wird das Signal direkt aus dem Muskel und unabhängig von äußeren Einflüssen abgeleitet [9]. Neben der Steuerung, die durch neue Technologien in der Signalextraktion und -verarbeitung, wie z.B. Mustererkennung, deutlich verbessert werden kann, wird auch die prothetische Anbin- 
dung an den Stumpf laufend optimiert $[10,12]$. Das Konzept der Osseointegration ermöglicht eine direkte Anbindung an das knöcherne Skelett mit bestmöglichem Erhalt des Bewegungsumfangs des Stumpfs [3]. Hier verhindert noch die Gefahr der Weichteil- und Knocheninfektionen aufgrund des perkutanen Durchtritts eine breite Anwendung.

\section{Diskussion}

Ein kompletter Funktionsverlust oder sogar die Amputation der oberen Extremität stellt für die betroffenen Patienten einschneidende und traumatische Erlebnisse dar. Diese gehen oft mit massiven Einbußen der Lebensqualität einher [7]. Der prothetische Ersatz, auch wenn er in manchen Fällen mit einer notwendigen elektiven Amputation verbunden ist, ermöglicht eine Reintegration ins Arbeitsund Sozialleben und somit eine verbesserte Lebensqualität.

Selektive Nerventransfers im Rahmen einer TMR-Operation bieten Patienten mit hohen Amputationen der oberen Extremität die Möglichkeit einer verbesserten und v. a. intuitiveren Prothesensteuerung. Gleichzeitig lassen sich vorhandene Neurome am Amputationsstumpf behandeln. Der prothetische Extremitätenersatz im Sinne der dargestellten bionischen Rekonstruktion ist ein neuer Ansatz in der Rehabilitation von Patienten, bei denen alle Möglichkeiten der biologischen Rekonstruktion ausgeschöpft wurden. Die adäquate Indikationsstellung ist sicherlich die größte Herausforderung und unterliegt dem Grundsatz „Primum non nocere“.

\section{Fazit für die Praxis}

\section{- Eine sinnvolle und erfolgreiche biologische Rekonstruktion soll und darf nicht mit dem prothetischen Ersatz konkurrieren. \\ - Zur Umsetzung der dargestellten Konzepte des prothetischen Ex- tremitätenersatzes bedarf es einer interdisziplinären Zusammenarbeit von Chirurgen, Physiotherapeuten, Technikern und Orthopädietechni- kern, aber auch Psychologen und Wissenschaftlern.}

- Der prothetische Extremitätenersatz ermöglicht eine funktionelle Wiederherstellung bei Patienten mit funktionsloser und asensibler Hand bzw. nicht funktionstüchtigem Arm nach massiven Weichteil- oder Nervenverletzungen sowie angeborenen Defekten.

Korrespondenzadresse
Univ.-Prof. Dr.
Oskar C. Aszmann
Klinische Abteilung für
Plastische und Rekonstruktive
Chirurgie, Christian Doppler
Labor für Wiederherstellung
von Extremitätenfunktionen
Universitätsklinik für
Chirurgie, Medizinische
Universität Wien
Währinger Gürtel 18-20,
1090 Wien, Österreich
oskar.aszmann@
meduniwien.ac.at

Funding. Open access funding provided by Medical University of Vienna.

\section{Einhaltung ethischer Richtlinien}

Interessenkonflikt. S. Salminger, J.A. Mayer, A. Sturma und O.C. Aszmann geben an, dass kein Interessenkonflikt besteht.

Dieser Beitrag beinhaltet keine von den Autoren durchgeführten Studien an Menschen oder Tieren. Für Bildmaterial oder anderweitige Angaben innerhalb des Manuskripts, über die Patienten zu identifizieren sind, liegt von ihnen und/oder ihren gesetzlichen Vertretern eine schriftliche Einwilligung vor.

Open Access Dieser Artikel wird unter der Creative Commons Namensnennung 4.0 International Lizenz (http://creativecommons.org/licenses/by/4.0/deed. de) veröffentlicht, welche die Nutzung, Vervielfältigung, Bearbeitung, Verbreitung und Wiedergabe in jeglichem Medium und Format erlaubt, sofern Sie den/die ursprünglichen Autor(en) und die Quelle ordnungsgemäß nennen, einen Linkzur Creative Commons Lizenz beifügen und angeben, ob Änderungen vorgenommen wurden.

\section{Literatur}

1. Aszmann OC et al (2010) Treatment of painful neuromas via end-to-side neurorraphy. Handchir Mikrochir Plast Chir 42(4):225-232

2. Aszmann OC et al (2015) Bionic reconstruction to restore hand function after brachial plexus injury: a case series of three patients. Lancet. https://doi. org/10.1016/s0140-6736(14)61776-1
3. Brånemark R, Brånemark PI, Rydevik $\mathrm{B}$, Myers RR (2001) Osseointegration in skeletal reconstruction and rehabilitation: a review. J Rehabil Res Dev 38(2):175-81.

4. Hruby LA et al (2017) Algorithm for bionic hand reconstruction in patients with global brachial plexopathies. J Neurosurg. https://doi.org/10. 3171/2016.6.jns16154

5. Kuiken TA et al (2004) The use of targeted muscle reinnervation for improved myoelectric prosthesis control in a bilateral shoulder disarticulation amputee. Prosthet Orthot Int 28(3):245-253

6. Leixnering $M$ et al (2013) Prevention of hand injuries-current situation in Europe. Handchir Mikrochir Plast Chir 45(6):339-343

7. Meyer TM (2003) Psychological aspects of mutilating hand injuries. Hand Clin 19(1):41-49

8. Oleske DM, Hahn JJ (1992) Work-related injuries of the hand: data from an occupational injury/ illness surveillance system. J Community Health 17(4):205-219

9. Pasquina PF et al (2014) First-in-man demonstration of a fully implanted myoelectric sensors system to control an advanced electromechanical prosthetic hand. J Neurosci Methods. https://doi. org/10.1016/j.jneumeth.2014.07.016

10. Roche AD et al (2014) Prosthetic myoelectric control strategies: a clinical perspective. Curr Surg $\operatorname{Rep} 2(3): 44$

11. Salminger $S$ et al (2015) Prosthetic reconstruction in high amputations of the upper extremity. Orthopade. https://doi.org/10.1007/s00132-0153113-0

12. Salminger $S$ et al (2016) Attachment of upper arm prostheses with a subcutaneous osseointegrated implant in transhumeral amputees. Prosthet Orthot Int 42:93

13. Salminger $S$ et al (2016) Improving arm function by prosthetic limb replacement in a patient with severe arthrogryposis multiplex congenita. JRehabil Med 48(8):725-728

14. von Schroeder HP, Botte MJ (1998) Crush syndrome of the upper extremity. Hand Clin 14(3):451-456

15. Souza JM et al (2014) Targeted muscle reinnervation: a novel approach to postamputation neuroma pain. Clin Orthop Relat Res. https://doi.org/10. 1007/s11999-014-3528-7

16. Sturma A et al (2015) A surface EMG test tool to measure proportional prosthetic control. Biomed Tech (Berl) 60(3):207-213 